InnOvaciOnes de NegOciOs 18(35):

(C) 2021 UANL, Impreso en México (ISSN: 2007-1191)

Recepción: 27 de noviembre de 2020 Aceptación: 7 de enero 2021

\title{
La estructura de concesiones del sector minero en México: un estudio de concentración y competitividad (The structure of concessions in the mining sector in Mexico: A study of concentration and competitivity)
}

\author{
Martha Nelly Garza Garza ${ }^{1}$ \& Jorge Omar Moreno Treviño 2 \\ Instituto Tecnologico de Estudios Superiores de Monterrey, EGADE Business \\ School 1; Universidad Autónoma de Nuevo León Facultad de Economia 2 \\ nellygarza@tec.mx 1, jorge.morenotr@uanl.edu.mx²
}

\begin{abstract}
Abstact: We analyze the concentration of concessions in the Mexican mining sector, as well as the degree of competition that this sector presents based on the holding of concessional hectares as of December 31, 2016. Our work finds that $13 \%$ of the hectares concessionaires belong to the business groups listed on the Mexican Stock Exchange (BMV), and the remaining $87 \%$ belong to many owners, both individuals and legal entities, identifying the main concessionaires, during the development of this research. Likewise, we found that despite being mostly people those who have mining concessions, with $85 \%$; it is the legal entities who have more hectares concessioned to their name, with $65 \%$ of the total. Finally, considering all mining concessionaires, we conclude there is a high inequality in the distribution of hectares (Gini coefficient), but on the other hand, there is no evidence that the concentration in said concessions configures a non-competitive market structure, of according to the Herfindahl and Hirschman Index $(\mathrm{IHH})$.
\end{abstract}

Keywords: Mining sector, competition, concentration, concessions.

JEL: L72, D4, K23.

Resumen: En la presente investigación se analiza la concentración de concesiones en el sector minero mexicano, así como el grado de competencia que este sector presenta en función de la tenencia de hectáreas concesionadas al 31 de diciembre de 2016. Nuestro trabajo encuentra que

\section{La estructura de concesiones del sector minero en México}


$13 \%$ de las hectáreas concesionadas pertenece a los grupos empresariales que cotizan en la Bolsa Mexicana de Valores (BMV), y el $87 \%$ restante pertenece a un amplio número de titulares, tanto personas físicas como personas morales, identificando a los principales concesionarios, durante el desarrollo de esta investigación. Asimismo, se encontró que a pesar de ser en su mayoría personas fisicas las que tienen concesiones mineras, con un $85 \%$; son las personas morales quienes tienen más hectáreas concesionadas a su nombre, con un $65 \%$ del total. Finalmente, considerando la totalidad de concesionarios mineros, se concluye que existe una alta desigualdad en la distribución de hectáreas (coeficiente de Gini), pero por contraparte, no hay evidencia de que la concentración en dichas concesiones configure una estructura de mercado no competitiva, de acuerdo con el Índice de Herfindahl e Hirschman $(\mathrm{IHH})$.

Palabras clave: Sector minero, competencia, concentración, concesiones.

\title{
Introducción
}

Según la Secretaría de Economía (SE) (2017a), la minería es uno de los principales sectores que contribuye en la economía nacional, aportando un $4 \%$ del Producto Interno Bruto (PIB). Además, este sector al mes de julio de 2015 generó 352 mil 666 empleos directos y más de 1.6 millones de empleos indirectos, de acuerdo con el Instituto Mexicano del Seguro Social (IMSS).

En el ámbito fiscal, el sector minero se ha tratado como un sector sensible por parte del gobierno para el establecimiento 0 incremento de contribuciones, debido a que su operación se considera de alto riesgo para sus trabajadores, además de que se requieren de fuertes inversiones de capital para poder operar un proyecto en este sector. Sin embargo, en la Reforma Fiscal que entró en vigor en el ejercicio 2014, se consideraron cambios importantes a este sector, destacando el establecimiento de nuevos derechos y la modificación de las reglas para la deducción de sus gastos preoperativos, pudiendo hacerlo hasta en diez ejercicios y no sólo en uno, como estaba planteado hasta el 2013.

En este sentido, el presente artículo tiene como finalidad analizar el grado de concentración en hectáreas que tienen los titulares de las

\author{
Garza, M. \& Moreno, J.
}


concesiones mineras, con la finalidad de cuantificar, identificar y evaluar las condiciones de desigualdad y concentración en el mercado, construyendo para este fin las bases de datos, en función de la información pública disponible. Para el análisis de la información se emplean los métodos: curva de Lorentz, coeficiente de Gini y Coeficiente de Hirschman-Hirshleifer (HHI).

En nuestro país, la Comisión Federal de Competencia Económica (COFECE) (2017) es la encargada de regular la forma de operar de los mercados, con la intención de garantizar la competencia económica y se castiguen o eliminen los monopolios, las concentraciones ilícitas, las barreras y otras restricciones al mercado. De acuerdo con esta Comisión, en el caso del sector minero, específicamente en los siguientes subsectores: 1) Minería de minerales metálicos y no metálicos, excepto petróleo y gas y 2) Servicios relacionados con la minería, no se encontraron concentraciones que frenen la estructura competitiva del mercado y se consideren no autorizadas de acuerdo con la Ley Federal de Competencia Económica (LFCE).

Por su parte, en México se han otorgado un gran número de permisos y concesiones mineras, lo que ha ocasionado, según Sariego (2009) que se presente el problema de la creación de un sector monopólico por parte de los empresarios mexicanos que cuentan con los recursos económicos para invertir en dichos proyectos, sin embargo, salvo los análisis de la COFECE en donde se descarta tal concentración, no existen estudios adicionales a este sector, que permitan conocer el grado de competencia existente.

Los grupos mineros mexicanos, que cotizan en la BMV, los cuales se analizan en este artículo con la intención de confirmar si muestran concentración en las hectáreas concesionadas son: Compañía Minera Autlán, SAB de CV, Grupo México, SAB de CV, Industrias Peñoles, SAB de CV y Minera Frisco, SAB de CV

Es importante mencionar que, esta investigación no vincula la producción minera extraída que realiza cada titular de las concesiones mineras, ni tampoco incluye si algún proyecto minero no está operando, lo anterior porque esta información no es pública ni accesible de manera directa, por lo tanto, el enfoque que se presenta utiliza solamente las dimensiones de las concesiones mineras como variable de desempeño en el mercado.

\section{La estructura de concesiones del sector minero en México}


Revisión de literatura

En esta sección enfatizaremos la referencia a la literatura aplicada al tipo de estudio que tenemos como objetivo realizar, esto es, medir directamente la concentración en concesiones, algo que para el

En el análisis de la estructura de una industria, se considera que es clave en el grado de concentración que guardan sus participantes en el mercado, puesto que según el paradigma Estructura-Conducta-Resultados citado por Bain (1951 y 1957) es la causa de los resultados de las empresas en los mercados (Furió y Alonso, 2008).

La concentración de mercado, según Furió, E. \& Alonso, M. (2008), se define como el grado en que el mercado a analizar está concentrado, según el número de agentes, en la mayoría de los casos considerados como productores o vendedores. Si lo explicamos de forma simple, sería que proporción de las ventas o de la producción son representados por los pocos y grandes productores en relación con la totalidad de la industria.

Para demostrar la importancia del estudio de la concentración de los mercados se plasman tres investigaciones: la primera de ellas analiza la banca comercial en México; la segunda investiga el mercado televisivo en América Latina y su influencia en las decisiones políticas de los ciudadanos, y la tercera, analiza la tendencia hacia la hiperconcentración del mercado de distribución de alimentos en nuestro país.

En una investigación para estudiar la concentración en el mercado de la banca comercial en México, Chavarín, R. (2012) señala que un conjunto de grupos económicos ha mantenido el interés en tener un banco comercial en su red de negocios. En ese artículo se presentaron cuáles son los bancos ligados a grupos económicos en México, señalando Chavarín, R. (2012) en un cuadro a los siguientes bancos (el año de operación) y su grupo económico:

-Afirme (1995) -Villacero;

- Ahorro Famsa (2007) -Famsa;

- Amigo (2007) -Grupo Inmobiliario Landus (en marzo de 2011 el banco fue comprado por ABC Holding y una subsidiaria del Grupo Cemex);

-Autofin México (2006) -Autofin;

- Azteca (2002) -Elektra -TV Azteca;

\section{Garza, M. \& Moreno, J.}


- Bancoppel (2007) -Coppel;

- Banorte (privatizado en 1992) -Gruma;

- Del Bajío (1995) -Grupo Soni;

- Fácil (2007) -Chedraui;

- Inbursa (1994) -Carso, América Móvil, Ideal;

- Invex (1994) -Liverpool;

- Interacciones (1993) -Hermes -Transportes;

- Ixe (1994) -Gigante, Xtra- Saba, Soriana (en noviembre de 2010, el banco fue comprado por Banorte);

- Multiva (2007) -Ángeles;

- Ve por más (2003) -Mexichem

Chavarín, R. (2012) establece que es a partir del 2008 donde se considera que los 15 bancos señalados anteriormente son los que representan entre el $10 \%$ y $20 \%$ de la concentración del sistema, utilizando el Índice de Herfindahl e Hirschman ( $(\mathrm{HH})$, medido en activos, cartera y captación totales. Asimismo, "el análisis permite inferir que el crecimiento de estos bancos, en especial de los pequeños, haría posible reducir los niveles de concentración que benefician a los grandes bancos de propiedad extranjera. Sin embargo, el crecimiento de estos bancos reforzaría ciertos problemas de incentivos y favorecería las operaciones de los grupos económicos a los que están ligados."

En otra investigación en donde se analizó la concentración del mercado televisivo en América Latina, Trejo. (2010) establece que la concentración de los medios de comunicación suele ser reconocida como un obstáculo a la pluralidad, sin embargo, en América Latina no existen límites legales a que se realice la concentración de muchos medios en pocas manos. En su trabajo Trejo (2010) examina como se acaparan los recursos comunicacionales en cuatro grandes grupos mediáticos y, utilizando el Índice de Herfindahl e Hirschman $(\mathrm{HHH})$ se establece el índice de concentración de la televisión en Argentina, Brasil, Chile, México y Venezuela. "Más adelante esos datos se comparan con indicadores de cultura política en tales países para determinar si hay relación entre la concentración de la televisión y la propagación de valores ciudadanos, como el aprecio por la democracia". Los efectos que puede alcanzar la concentración de los medios de comunicación cobran

\section{La estructura de concesiones del sector minero en México}


especial relevancia en América Latina, en donde "el arraigo que tienen las empresas comunicacionales dentro del entramado político de cada país y debido, naturalmente, a la influencia que alcanzan en la conformación de convicciones y decisiones cívicas entre los ciudadanos. Si el derecho a la comunicación se encuentra estrechamente relacionado con el acceso de la sociedad a los medios, entonces la concentración de los medios constituye, cada vez más, un impedimento fundamental para una plena expansión de la democracia".

En los mercados como el de distribución de alimentos en México, algunos trabajos han resaltado la tendencia del modelo económico encaminándose hacia la hiperconcentración de capital comercial en pocas empresas, así como la capacidad para lograr una amplia expansión en zonas metropolitanas y ciudades de todo el país. Asimismo, han flexibilizado el tamaño de los establecimientos para diversificar su localización a distintos estratos de ingreso y consolidar estrategias de penetración. (Gasca, J. \& Torres, F., 2014). En particular, las principales cadenas de supermercados que destacan en el estudio señalado anteriormente por su grado de concentración en la distribución alimentaria en México son: Wal-Mart, Soriana, Comercial Mexicana y Chedraui.

Las investigaciones anteriores son ejemplos de la importancia del estudio de la concentración de los mercados, en donde se muestra como herramienta de análisis de la competencia y permite, a los expertos del sector, plantear modelos de negocios que logren enfrentar a sus competidores dominantes.

Según Grullon, Larkin y Michaely (2017), citados por Núñez y De Furquim (2018), señalan que "existe evidencia en la literatura sobre la correlación entre aumentos en la concentración del mercado de productos y mayores márgenes de ganancia, retornos positivos anormales de acciones y acuerdos de fusiones y adquisiciones (F\&A) más rentables, los datos financieros de las empresas pueden ser una herramienta muy potente para analizar la competencia en la economía digital."

Considerando las investigaciones anteriormente señaladas. y debido a la relevancia que representan los estudios de concentración de los mercados y a la falta de análisis sobre este tema en el sector minero es que se presenta este trabajo, en donde se muestra el acaparamiento en hectáreas que tienen

\author{
Garza, M. \& Moreno, J.
}


los titulares de las concesiones mineras, con la finalidad de cuantificar, identificar y evaluar el grado de concentración que tienen, construyendo para este fin las bases de datos, en función de la información pública disponible. Es importante mencionar que, esta investigación no vincula la producción minera extraída que realiza cada titular de las concesiones mineras, ni tampoco incluye si algún proyecto minero no está operando, lo anterior porque esta información no es pública ni accesible de manera directa, por lo tanto, el enfoque que se presenta utiliza solamente las dimensiones de las concesiones mineras como variable de desempeño en el mercado.

Construcción de datos

Para el desarrollo de esta investigación se construyó una base de datos a partir del banco de información "Cartografía de concesiones mineras en el territorio nacional" publicada por la Secretaría de Economía (SE., 2017b), con información actualizada al 31 de diciembre de 2016.

Para hacer la clasificación de las empresas, plantas y minas que pertenecen a cada grupo que cotiza en la BMV, se creó una base de datos a partir del Directorio del Sector Minero, que se encuentra en la página de la Secretaría de Economía (SE, 2017c).

El número de títulos que otorgan concesiones mineras en México es de 25,554 . Estos títulos se encuentran respaldados por 22,052,681 hectáreas. Según el INEGI (2017), la superficie continental de nuestro país es de $1,960,189 \mathrm{~km} 2$, lo que representa que se encuentra concesionada un $11.25 \%$.

Del análisis de la información, se encontró que las 6 entidades federativas con mayor número de hectáreas concesionadas son: Sonora $(18.12 \%)$, Chihuahua $(9.15 \%)$, Durango $(9.01 \%)$, Coahuila $(7.70 \%)$, Zacatecas $(7.65 \%)$ y Jalisco $(7.37 \%)$. Así también, se identificaron y clasificaron los titulares de las concesiones vigentes al 31 de diciembre de 2016, en personas físicas y personas morales, considerando el número de hectáreas concesionadas, el número de títulos y de titulares que las concentran, obteniendo la relación que se muestra en el Cuadro 1.

\section{La estructura de concesiones del sector minero en México}


Cuadro 1. Titulares de las concesiones mineras

\begin{tabular}{lcccccc} 
Titular & Hectáreas & $\%$ & Títulos & $\%$ & Titulares & $\%$ \\
\hline Persona Moral & $14,430,413$ & 65 & 11,205 & 44 & 1,286 & 15 \\
Persona Física & $7,622,267$ & 35 & 14,349 & 56 & 7,250 & 85 \\
\cline { 2 - 2 } Total & $22,052,681$ & & 25,554 & & 8,536 &
\end{tabular}

Fuente: Elaboración propia con datos de la Secretaría de Economía

De acuerdo con la tabla anterior, a pesar de ser en su mayoría personas físicas las que tienen la propiedad de las concesiones mineras, con un 85\%; son las personas morales quienes tienen más hectáreas concesionadas a su nombre, con un $65 \%$ del total.

Es importante resaltar que el número de hectáreas concesionadas en cada título es variable, teniendo concesiones desde menos de .0001 hectáreas, hasta un título en Jalisco con una superficie de 231,556.16 hectáreas.

A continuación, se realiza un análisis pormenorizado, donde se incluyen las 20 personas morales y las 20 personas físicas que mayor número de hectáreas concesionadas tienen, así como la concentración de hectáreas que tienen las empresas de los grupos que cotizan en la BMV. En el Cuadro 2, se muestra el análisis correspondiente a las 20 personas morales que mayor número de hectáreas tienen concesionadas.

Es importante destacar que, dentro de esta tabla se encuentran empresas que pertenecen a los grupos que cotizan en la BMV, las cuales fueron identificadas en el Cuadro 3.

Posteriormente, en el Cuadro 4 se refleja el total de hectáreas y títulos que tienen concesionados las empresas que conforman cada uno de los grupos que cotizan en la BMV, identificando que sólo el $13 \%$ del total de hectáreas concesionadas a la minería pertenece a estos grupos.

Finalmente, en el Cuadro 5 se analiza la participación que tienen las personas físicas en el sector minero, destacando a las principales 20 , que mayor número de hectáreas tienen concesionadas. Es importante resaltar que la información que vincula producción minera y tenencia de las concesiones mineras no es pública ni accesible de manera directa, por tanto, nuestro enfoque en esta investigación utiliza solamente las características de esta última como variable de desempeño en el mercado.

\section{Garza, M. \& Moreno, J.}


Cuadro 2. Las "20" Personas Morales que tienen mayor cantidad de hectáreas en concesiones mineras

\begin{tabular}{lll} 
& Hectárea & Título \\
Persona Moral & s & s \\
\hline Exploraciones Mineras Parreña, S.A. de C.V. & 836,715 & 525 \\
Minerales Monclova, S.A. de C.V. & 564,217 & 115 \\
Minera del Norte, S.A. de C.V. & 527,967 & 140 \\
Compañía Minera La Parreña, S.A. de C.V. & 400,400 & 541 \\
Minera Penmont, S. de R.L. de C.V. & 376,652 & 193 \\
Minera Carbonífera Rio Escondido, S.A. de C.V. & 331,947 & 32 \\
Canplats de México, S.A. de C.V. & 323,278 & 8 \\
Industrial Minera México, S.A. de C.V. & 310,120 & 531 \\
Minera María, S.A. de C.V. & 236,381 & 110 \\
Las Encinas, S.A. de C.V. & 235,682 & 66 \\
Minera Plata Real, S. de R.L. de C.V. & 225,072 & 20 \\
Minera Teck, S.A. de C.V. & 197,678 & 14 \\
Exmin, S.A. de C.V. & 180,581 & 34 \\
Compañía Minera Pangea, S.A. de C.V. & 172,393 & 24 \\
Grupo Ontiveros de Minerales de México, S. de R.L. de & 169,355 & 4 \\
C.V. & 161,608 & 122 \\
Exploraciones Mineras Peñoles, S.A. de C.V. & 156,001 & 26 \\
Minera Teocuitla, S.A. de C.V. & 136,527 & 81 \\
Compañía Fresnillo, S.A. de C.V. & 136,100 & 9 \\
Minera Bhp Billiton, S.A. de C.V. & 135,338 & 110 \\
\hline Minera Real de Ángeles, S.A. de C.V. & $\mathbf{5 , 8 1 4 , 0 1}$ & \\
& $\mathbf{2}$ & $\mathbf{2}$ \\
& & \\
& &
\end{tabular}

Fuente: Elaboración propia con datos de la Secretaría de Economía. 
Cuadro 3. Personas Morales que tienen mayor cantidad de hectáreas en concesiones mineras pertenecientes a los Grupos que cotizan en la BMV Grupo

que cotiza en la BMV Industria s Exploraciones Mineras Parreña, S.A. de Peñoles C.V. Hectárea Título Industria $S$ Peñoles Compañía Minera La Parreña, S.A. de C.V. 400,400 541 Grupo México Industrial Minera México, S.A. de C.V. $\quad 310,120 \quad 531$ Minera

Frisco Minera Real de Ángeles, S.A. de C.V. 135,338 110 Fuente: Elaboración propia con datos de la Secretaría de Economía.

Cuadro 4. Hectáreas concesionadas a los Grupos Mineros que cotizan en la

\begin{tabular}{lll}
\multicolumn{3}{c}{$B M V$} \\
\hline & Hectáreas & Títulos \\
\cline { 2 - 3 } Compañía Minera Autlán & 73,475 & 213 \\
Grupo México & 577,397 & 681 \\
Industrias Peñoles & $1,505,380$ & 1,318 \\
Minera Frisco & $\mathbf{7 3 9 , 8 3 9}$ & 820 \\
\cline { 2 - 3 } Total & $\mathbf{2 , 8 9 6 , 0 9 0}$ & $\mathbf{3 , 0 3 2}$ \\
& & \\
Total de Concesiones & $22,052,681$ & 25,554 \\
Proporción & $\mathbf{1 3 \%}$ & $\mathbf{1 2} \%$ \\
\hline
\end{tabular}

Fuente: Elaboración propia con datos de la Secretaría de Economía. 
Cuadro 5. Las "20" Personas Físicas que tienen mayor cantidad de hectáreas en concesiones mineras

\begin{tabular}{|c|c|c|}
\hline Persona Física & Hectáreas & Títulos \\
\hline JJA & 580,305 & 8 \\
\hline IABR & 350,451 & 16 \\
\hline CNRJ & 255,517 & 14 \\
\hline JIRG & 161,572 & 42 \\
\hline GGNH & 110,192 & 14 \\
\hline JCAN & 108,429 & 3 \\
\hline RMA & 101,490 & 8 \\
\hline JCSS y Socios & 97,476 & 10 \\
\hline OPQC & 88,128 & 3 \\
\hline ANA y Socios & 84,742 & 6 \\
\hline GRF & 80,520 & 3 \\
\hline CNRJ y Socios & 72,898 & 2 \\
\hline FABT & 65,611 & 4 \\
\hline EIM & 61,583 & 12 \\
\hline JLGA & 53,009 & 5 \\
\hline FJAT & 51,119 & 2 \\
\hline ARV y Socios & 50,767 & 2 \\
\hline OPE & 49,862 & 4 \\
\hline VDCB & 48,823 & 1 \\
\hline \multirow[t]{2}{*}{ HEME } & 47,931 & 1 \\
\hline & $2,520,425$ & 160 \\
\hline
\end{tabular}

Nota: por respeto a la confidencialidad, se presentan únicamente las iniciales de las personas físicas enlistadas en la publicación oficial.

Fuente: Elaboración propia con datos públicos de la Secretaría de Economía 


\section{Metodología}

La investigación utiliza como herramientas para el procesamiento de datos financieros a la curva de Lorentz, el Coeficiente de Gini y el Índice de Herfindahl e Hirschman (IHH).

La curva de Lorentz en una representación gráfica que muestra la distribución de una variable entre los sujetos de estudio. Para efectos de esta investigación, se considerará como variable a analizar, el número de hectáreas que se encuentran concesionadas a los sujetos de estudio y se revisará si la distribución de estas es equitativa entre los particulares.

El coeficiente de Gini va de la mano con la curva de Lorentz, puesto que el indicador que mide el área de la curva de Lorentz, y demuestra si existe 0 no desigualdad entre los sujetos. El valor que toma fluctúa entre 0 y 1 ; si el indicador se acerca a 0 , señala que existe igualdad en la distribución entre los sujetos; si el indicador se aproxima a 1, señala una desigualdad existente, lo que puede conllevar a tener concentración de riqueza.

La fórmula para el cálculo del coeficiente de Gini es la siguiente:

$$
G=\left|1-\sum_{k=1}^{k=n-1}\left(X_{k+1}-X_{k}\right)\left(Y_{k+1}+Y_{k}\right)\right|
$$

Donde las variables muestran: " $X$ " la proporción acumulada de la población, y "Y" muestra la proporción acumulada de los ingresos de la población, y "n" es el número de participantes.

El coeficiente de Gini se ha utilizado por el Consejo Nacional de Evaluación de la Política de Desarrollo Social (CONEVAL) para medir la pobreza en México, mostrando la desigualdad existente en los ingresos que obtienen las personas.

En la presente investigación, se sustituye la variable "y" por el número de hectáreas mineras que se encuentran concesionadas a los titulares. Es a través del Coeficiente de Gini que se revisa si la distribución de estas es equitativa entre los particulares, o bien, se mide el grado de desigualdad que existe en la distribución de dichas hectáreas.

Adicionalmente, la concentración de poder en el mercado se medirá con el Índice de Herfindahl e Hirschman (IHH). Según Flores (2006), la

\section{Garza, M. \& Moreno, J.}


concentración se emplea en los estudios económicos para medir la estructura de los mercados, por lo que considera el número y tamaño que tienen las empresas en un mercado determinado. El valor que toma el IHH fluctúa de 0 a 10,000. A medida que se incrementa el citado índice significa que se incrementa la concentración en pocos particulares.

La fórmula del Índice de Herfindahl e Hirschman $(\mathrm{IHH})$ es la siguiente:

$$
H=\sum_{i=1}^{n} S_{i}^{2}
$$

Donde el citado índice "H" se calcula a través de la suma de los cuadrados del número de empresas en el mercado que lo componen.

El índice de Herfindahl e Hirschman $(\mathrm{IHH})$ se utiliza como indicador de análisis en los mercados para revisar si la concentración entre los titulares refleja la configuración de un monopolio u oligopolio, o también, para medir la diversificación de un mercado financiero.

En la presente investigación se utiliza el Índice de Herfindahl e Hirschman $(\mathrm{HH})$ como el indicador empleado para medir el grado de concentración en hectáreas que tienen los concesionarios en el sector minero, y confirmar si existe evidencia que demuestre la conformación de una estructura de mercado no competitiva.

\section{Estimación y resultados}

Para realizar la estimación de los indicadores, se utiliza la base de datos construida a partir del banco de información "Cartografía de concesiones mineras en el territorio nacional" publicada por la Secretaría de Economía (SE., 2017b), con información actualizada al 31 de diciembre de 2016.

Para calcular la curva de Lorentz y poder realizar la determinación del coeficiente de Gini se concentraron en 2 archivos los titulares de las concesiones mineras, si eran personas físicas, o personas morales. Posteriormente, se ordenaron el número de hectáreas concesionadas por titular en orden ascendente, obteniendo el porcentaje que representan de la totalidad.

\section{La estructura de concesiones del sector minero en México}


En la curva de Lorentz es necesario estimar 2 líneas: en la primera de ellas, considerando la sumatoria, en orden ascendente, de las proporciones que tienen los titulares; y en la otra línea, considerando la sumatoria, como si todos tuvieran una misma participación. Para determinar el coeficiente de Gini, y plasmar la curva de Lorentz, las variables que se consideraron son: " $X$ " muestra proporción acumulada de los titulares concesionarios (divididos entre personas físicas y personas morales) y "Y" por la proporción acumulada de las hectáreas concesionadas

Para determinar dicho coeficiente, y poder reflejar lo que mide el área de la curva de Lorentz en la gráfica, después de ordenar el número de hectáreas concesionadas por titular en orden ascendente, y obtener el porcentaje de la proporción que representan, es necesario ir sumando cada proporción de los titulares, en forma acumulativa, para posteriormente, multiplicarlo por la sumatoria acumulativa de las proporciones, considerando que todos los titulares tienen el mismo número de hectáreas.

Para la determinación del Índice de Herfindahl e Hirschman $(\mathrm{IHH})$ se hizo de la misma forma que el coeficiente de Gini, concentrando en 2 archivos, los titulares de las concesiones mineras, si son personas físicas, o personas morales. Posteriormente, se ordenaron el número de hectáreas concesionadas por titular en orden ascendente, sólo que se obtuvo el cuadrado de la proporción que tienen.

La figura 1 muestra la curva de Lorentz que se obtiene para las concesiones mineras, donde el titular es una persona moral, sin importar si pertenece o no, a un grupo que cotiza en la BMV.

Garza, M. \& Moreno, J. 
Figura 1. Curva de Lorentz-Personas Morales en General

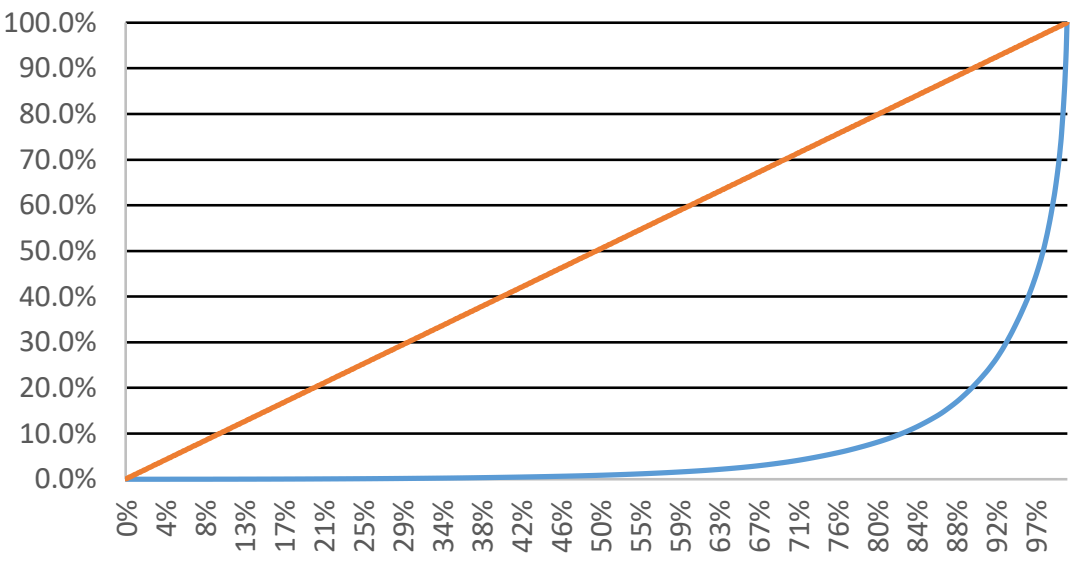

Fuente: Elaboración propia con datos de la SE.

Al revisar las figuras con las curvas de Lorentz y los coeficientes de Gini que se obtuvieron en las personas morales, podemos afirmar que existe una marcada desigualdad en el tamaño de las hectáreas que se han concesionado a estos sujetos. El coeficiente de Gini que se obtiene a partir de estos resultados es de 0.8715 , el cual, si lo apreciamos como porcentaje, se puede considerar que se presenta un $87.15 \%$ de dicha desigualdad.

En relación con las personas físicas, la figura 2 muestra la curva de Lorentz que tienen, considerando el número de hectáreas concesionadas a cada titular.

\section{La estructura de concesiones del sector minero en México}


Figura 2. Curva de Lorentz-Personas Físicas

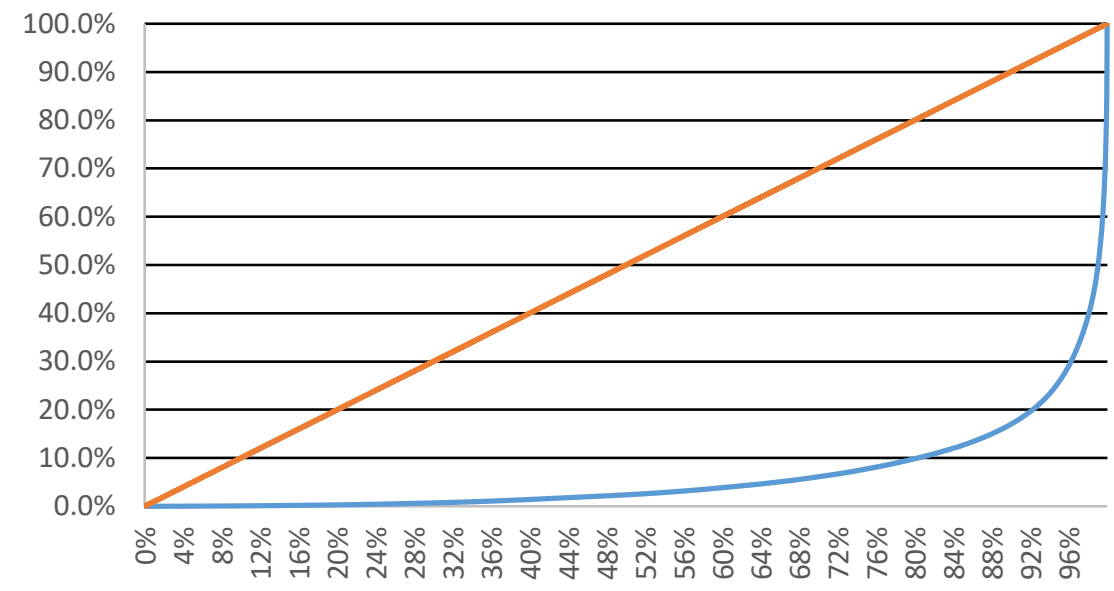

Fuente: Elaboración propia con datos de la SE.

De acuerdo con la figura anterior y el coeficiente de Gini obtenido es de 0.8748 , podemos afirmar que también para las personas físicas, existe una marcada desigualdad en las hectáreas que se les tienen concesionadas, incluso un poco mayor que la de las personas morales. Si lo apreciamos como porcentaje, se puede considerar que se presenta un $87.48 \%$ de dicha desigualdad.

Finalmente, en la tabla 6 se incluyen los resultados obtenidos del Índice de Herfindahl e Hirschman (IHH) que se obtuvieron en la investigación, tanto para los concesionarios que son personas físicas, como para aquéllos que son personas morales.

El $(\mathrm{IHH})$ calculado muestra que no existe evidencia de que exista concentración que configure una estructura de mercado no competitiva en dichas concesiones, es decir, el número de concesionarios es tan amplio y considerando el número de hectáreas mineras que cada uno puede explotar, no se consolida la concentración, permitiendo que exista una competencia real al igualar el juego entre los productores mineros, y facilitando a los consumidores para que tengan más opciones para adquirir los productos mineros.

\section{Garza, M. \& Moreno, J.}


Cuadro 6. Índice de Herfindahl e Hirschman (IHH)

\begin{tabular}{ll}
\hline & IHH calculado \\
\cline { 2 - 2 } Personas Morales & 130.47 \\
Personas Físicas & 120.37 \\
\hline
\end{tabular}

Fuente: Elaboración propia con datos de la Secretaría de Economía.

La perspectiva de "no configuración de una estructura de mercado no competitiva" en las concesiones mineras podría cambiar si se utiliza como variable de desempeño del mercado a la producción minera extraída que realiza cada titular de las concesiones, sin embargo, esta información no es pública ni accesible de manera directa, por lo tanto, el enfoque que le dimos utiliza solamente las características de las concesiones. Los resultados muestran en general una mayor concentración en las hectáreas concesionadas por parte de las personas morales que de las personas físicas.

\section{Conclusiones}

En la presente investigación se analizó la concentración de las concesiones en el sector minero en México. La construcción de datos involucró analizar datos puntuales a nivel documental.

La evidencia analizada en este artículo permite concluir que, considerando la totalidad de concesionarios mineros, hay evidencia de que existe una alta desigualdad en la distribución de hectáreas entre los mismos (coeficiente de Gini), pero por contraparte, no existe concentración en dichas concesiones, que logre configurar una estructura de mercado no competitiva, de acuerdo con el Índice de Herfindahl e Hirschman $(\mathrm{IHH})$.

Es importante aclarar que esta investigación no vincula la producción minera extraída que realiza cada titular de las concesiones mineras, ni tampoco incluye si algún proyecto minero no está operando, lo anterior porque esta información no es pública ni accesible de manera directa, por lo tanto, el enfoque que le dimos utiliza solamente las características de las concesiones mineras como variable de desempeño en el mercado.

\section{La estructura de concesiones del sector minero en México}




\section{Referencias}

Chavarín, R. (2012). Bancos ligados a grupos económicos en México y concentración de mercado en la banca comercial. Paradigma económico, 4(1). 5-26. Disponible en: https://biblat.unam.mx/es/revista/paradigma-economico/articulo/bancos-ligados-agrupos-economicos-en-mexico-y-concentracion-de-mercado-en-la-banca-comercial.

Comisión Federal de la Competencia Económica (2017). Concentraciones. Disponible en: https://www.cofece.mx/cofece/index.php/cofece/que-hacemos/concentraciones

Flores, D. (2006). Calidad y Concentración de Mercado en la Banca Comercial. Ensayos Revista de Economía, 25(1). 21-30. Disponible en: $\mathrm{http} / / /$ www.economia.uanl.mx/revistaensayos/xxv/1/C acion_de_Mercado.pdf

Furió, E. \& Alonso, M. (2008). Concentración Económica. Algunas consideraciones sobre su naturaleza y medida. Boletín Económico de Información Comercial Española, 2947. Disponible en: https://dialnet.unirioja.es/servlet/articulo?codigo=2707111 [consultado en mayo de 2019].

Gasca, J. \& Torres, F. (2014). El control corporativo de la distribución de alimentos en México. Revista Problemas del Desarrollo, 176(45). Disponible en: http://www.scielo.org.mx/scielo.php?script=sci_arttext\&pid=S030170362014000100007 [consultado en mayo de 2019].

Instituto Nacional de Estadística y Geografía (2017). Cuéntame. Disponible en: http://www.cuentame.inegi.org.mx/territorio/extension/default.aspx?tema=T

Núñez, G. \& De Furquim, J. (2018). La concentración de los mercados en la economía digital. Comisión Económica para América Latina y el Caribe. Disponible en: https://repositorio.cepal.org/bitstream/handle/11362/43631/1/S1800551_es.pdf [consultado en mayo de 2019].

Sariego, J. (2009). De minas, mineros, territorios y protestas sociales en México: los nuevos retos de la globalización. Cahiers des Amériques latines, 60(61), 173-192. Disponible en: http://cal.revues.org/1435

Secretaría de Economía, Gobierno de México. (2017). Directorio del Sector Minero Disponible en: http://www.desi.economia.gob.mx/empresas/

Secretaría de Economía, Gobierno de México. (2017). Minería. Disponible en: http://www.gob.mx/se/acciones-yprogramas/mineria

Trejo, R. (2010). Muchos medios en pocas manos: concentración televisiva y democracia en América Latina. Intercom: Revista Brasileira de Ciências da Comunicação. São Paulo, 33(1). 17-51. Disponible en: https://rtrejo.files.wordpress.com/2011/03/muchosmedios-en-pocas-manos-intercom-vol-33-no-1-2010.pdf

\section{Garza, M. \& Moreno, J.}

